\title{
Simulation Results and a Proof-of-Concept Implementation of the FleetNet Position-Based Router
}

\author{
Extended Abstract
}

\author{
Hannes Hartenstein ${ }^{1}$, Holger Füßler ${ }^{2}$, Martin Mauve ${ }^{2}$, and Walter Franz ${ }^{3}$ \\ 1 NEC Europe Ltd. Network Laboratories \\ D-69115 Heidelberg, Germany \\ hannes.hartenstein@ccrle.nec.de \\ 2 University of Mannheim \\ Department of Mathematics and Computer Science \\ D-68131 Mannheim, Germany \\ \{fuessler, mauve\}@informatik . uni-mannheim.de \\ 3 DaimlerChrysler Research and Technology \\ D-89081 Ulm, Germany \\ walter.franz@daimlerchrysler.com
}

\section{Introduction}

The FleetNet project 1 [1.23] develops a platform for inter-vehicle communications based on ad hoc networking principles. With an ad hoc network established by moving vehicles, a realm of applications - ranging from exchange of emergency warnings or sensor data to unicast communication between passengers of different vehicles and to integration of the vehicular ad hoc network with the Internet through static or mobile FleetNet gateways - could be enabled in a low-latency, robust and low-cost fashion.

Vehicular ad hoc networks show a high level of mobility and, correspondingly, a high degree of dynamics with respect to topological changes. Therefore, multi-hop communication represents a serious challenge. Since in a vehicular ad hoc network communication end-points might be addressed by specifying a geographic region (geocast) in addition to standard IP addressing, positional information has to be used in any case for forwarding packets. GPS (and in the future Galileo) receivers will soon become a standard piece of equipment in vehicles and can be used to support routing tasks. In previous publications we have shown on the basis of simulations that routing methods that make use of geographic information can outperform other routing approaches in the case of vehicular ad hoc networks. Example results will be given in Section 2.

As a 'proof-of-concept' as well as to compare simulation results with reality, we currently have set up a real-world test network consisting of $6 \operatorname{Smart}^{T M}$ cars equipped with GPS receivers, IEEE 802.11b wireless LAN NICs, and our FleetNet router. In this extended abstract we outline the testbed architecture of this 'FleetNet Demonstrator'

\footnotetext{
${ }^{1}$ The FleetNet Project is partly funded by the German Ministry of Education and Research (BMBF) under contract no. 01AK025. Partners are DaimlerChrysler AG, NEC Europe Ltd., Siemens AG, Robert Bosch GmbH, Temic GmbH, and the Universities of Mannheim, Braunschweig, Hannover and Hamburg-Harburg.
} 


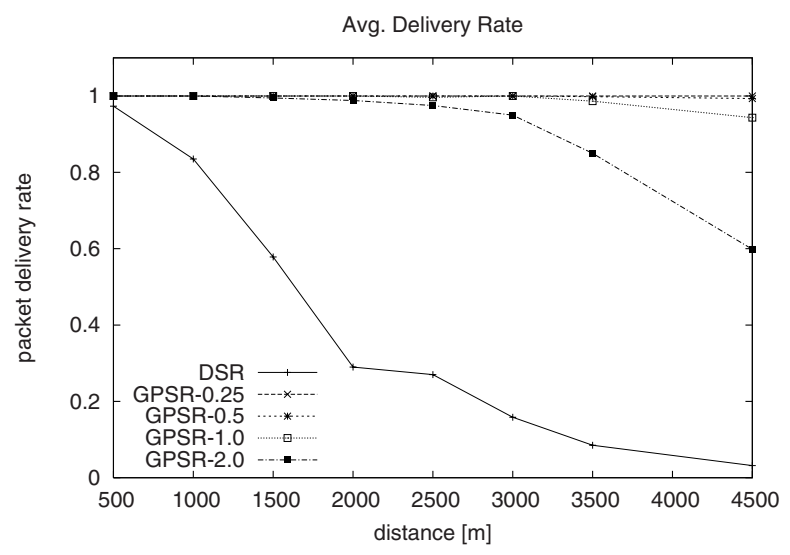

Fig. 1. A comparative study via NS-2 simulations of position-based routing (greedy part of greedy perimeter stateless routing, GPSR) and DSR. Shown is the achieved packet delivery ratio versus the multi-hop communication distance for the case of a highway scenario. The transmission range was set to $250 \mathrm{~m}$. The beaconing interval was set to $0.25,0.5,1.0$, and 2.0 seconds, respectively.

(FND) on both, hardware and software side and some first insights and experiences with the demonstrator in Section 3 and 4. The goal of this extended abstract is to present a current snapshot of the project with respect to position-based routing. A final evaluation of the FleetNet Demonstrator is underway. Related work has studied a real-world Dynamic Source Routing-based vehicular ad hoc test network [4] and single-hop characteristics with two vehicles [5].

\section{Simulation Results}

In position-based unicast routing, a packet header does not only indicate the destination's ID, e.g., IP address, but also the destination's approximate current geographic position. To acquire a destination node's position, a location service (see [6]) is used. As location service we use a the reactive location service (RLS) [7] that essentially has translated the DSR route discovery procedure to the domain of position-based ad hoc routing. At each hop packets are then forwarded in a greedy manner to the neighbor that is closest to the indicated destination's position. Each node is aware of its neighbors within transmission range through periodic 'beacon' messages. Several metrics for greedy routing as well as recovery strategies for failures of greedy routing are presented in [6]. The main advantage of position-based routing over classical topological approaches is given by the fact that there is no need for route setup and maintenance. Instead, packets are forwarded 'on the fly'. Thus, position-based routing deals very well with mobility. To 'prove' this claim, we have conducted simulation experiments based on realistic vehicle movements on a highway and a city scenario. In [8] we studied highway scenarios and showed that position-based routing outperforms Dynamic Source Routing (DSR) due to better handling of mobility and smaller header sizes (see Figure 11). In [9] we studied city 


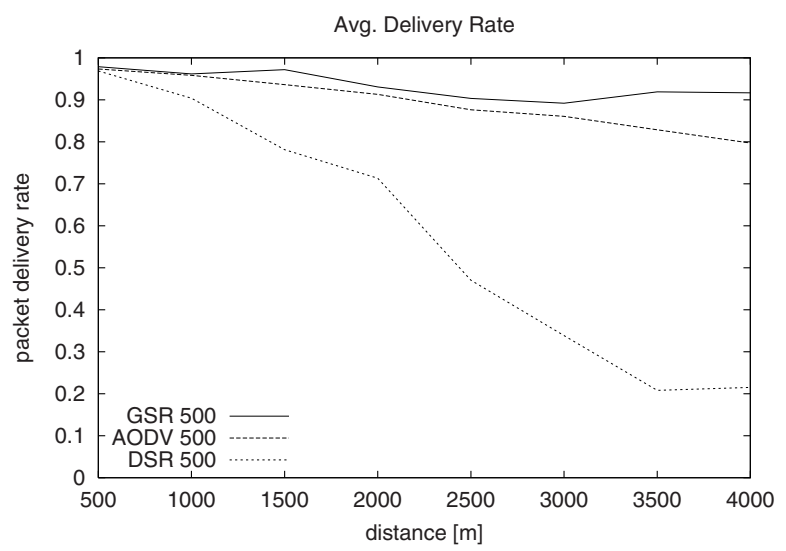

Fig. 2. A comparative study via NS-2 simulations of position-based routing making use of digital maps, DSR and AODV. Shown is the achieved packet delivery ratio versus the multi-hop communication distance for the case of a city scenario. The transmission range was set to $500 \mathrm{~m}$.

scenarios and compared position-based routing with DSR and with Ad Hoc On-Demand Distance Vector routing (AODV) showing that position-based routing outperforms the other approaches when a digital map of the city is available (see Figure 2). However, without a digital map position-based routing in a city environment suffers severely from the fact that frequently no greedy route is available to the destination and recovery strategies might fail due to radio obstacles.

\section{Testbed and Implementation Architecture}

In the 'real-world' test network, each of the six $\operatorname{Smart}^{T M}$ cars is equipped with a Linuxbased FleetNet router that forwards packets coming from either the 802.11b network interface card (NIC) or from the FleetNet car area network (FCAN), see Figure 3 a. We use external planar antennae with a gain of $4 \mathrm{dBi}$. Connected to the FCAN is a GPSreceiver with a navigational system. To support global monitoring of the 802.11-based ad hoc network, each car is in addition equipped with a GPRS NIC (Figure $3 \mathrm{~b}$ ).

The router is implemented as a user-space daemon that uses packet sockets in promiscuous mode on the wireless side. The FleetNet routing layer represents a '2.5 layer' approach by putting the FleetNet routing header between 802.11 MAC header and IP header (Figure 4). In order not to grep all packets coming from the FCAN, netfilter is used to select packets destined for other car subnets requiring wireless transport. For IP packets sent by the FCAN and addressed to another vehicle, a location service is used to determine the current geographic position of the vehicle corresponding to the destination IP address in case the position is not known. As location service we use again the reactive location service (RLS) [7]. When a required position is not known, a location request is flooded through the network. Corresponding packets for this destination are queued until the location reply is received. The forwarding decisions of the router 


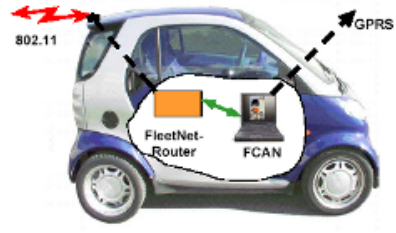

(a)

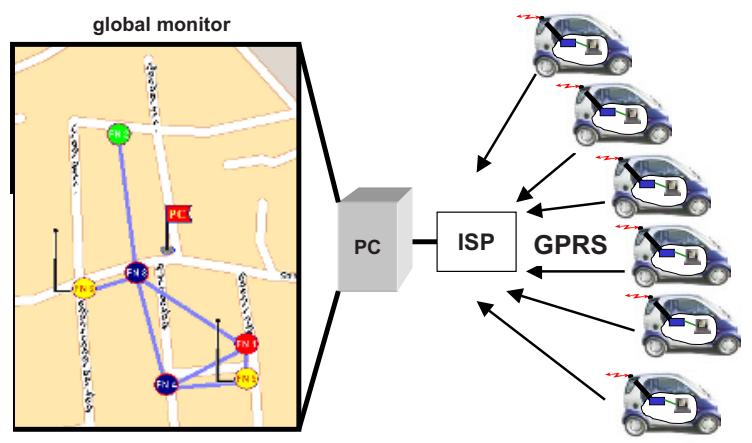

(b)

Fig. 3. (a) Each Smart car is equipped with a Linux-based FleetNet Router connected to the FleetNet car area network; (b) A global view of the ad hoc network is generated by having each car sending its local state via GPRS to a global monitor server.

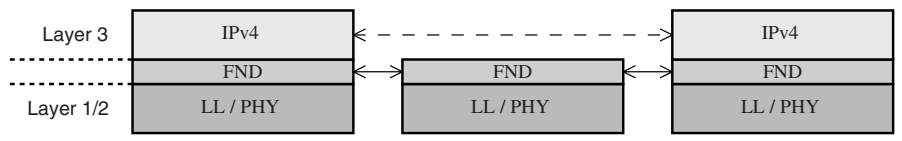

Fig. 4. The 2.5 layer approach taken in the 'proof-of-concept' implementation.

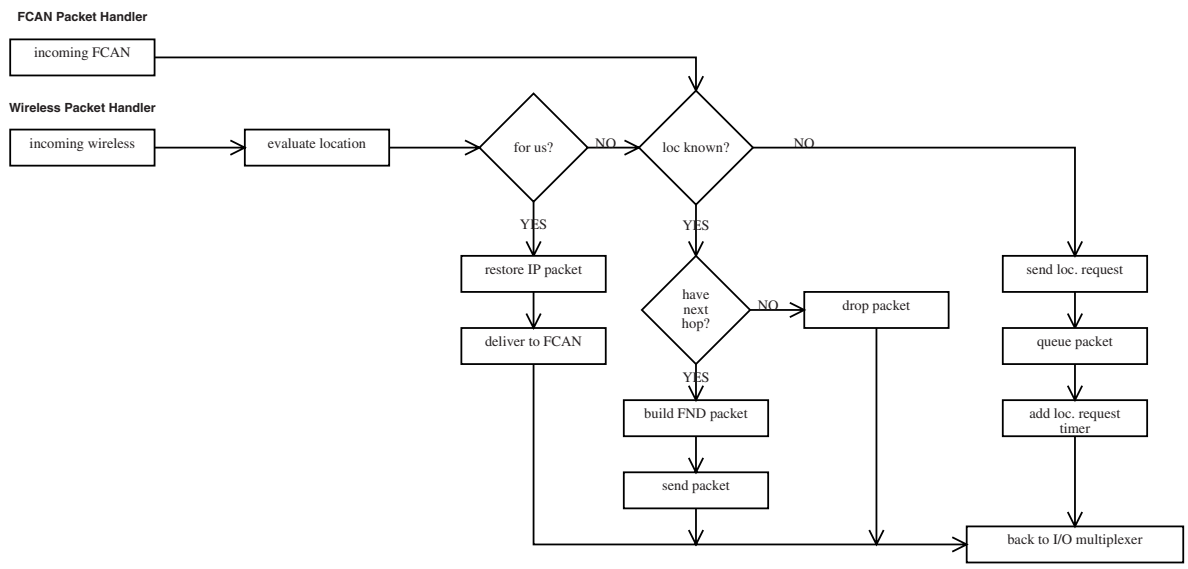

Fig. 5. Unicast position-based routing logic.

are based on greedy position-based routing as outlined in [10]: a packet is given to the neighbor that is closest to the final destination's position. Information on the positions of the neighbors is gained through periodic beacons each node sends out as one-hop broadcasts. The routing logic including location requests/replies is given in Figures 5 and 6 


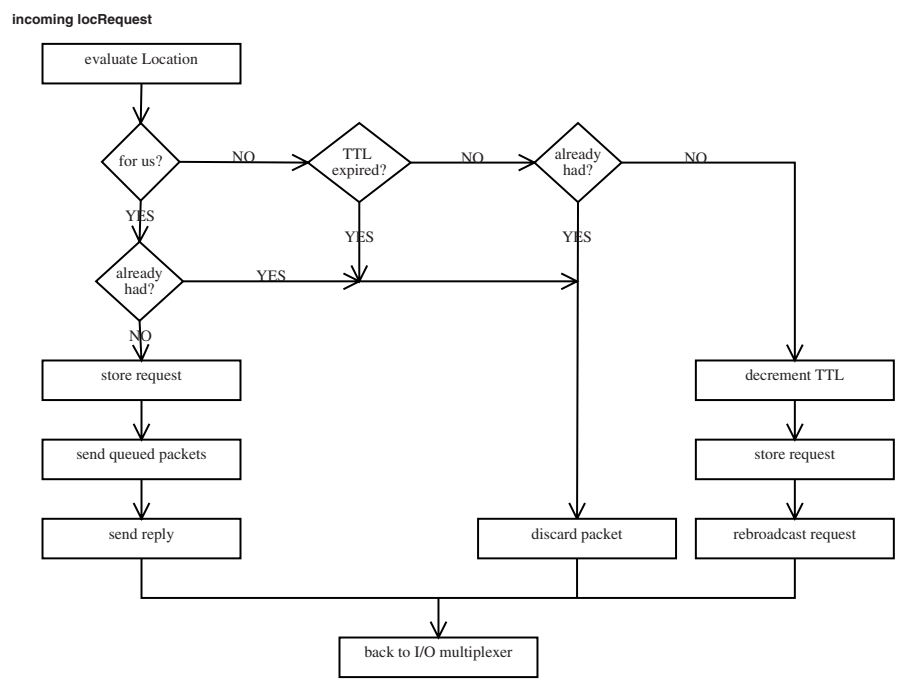

Fig. 6. Handling of location request packets.

\section{First Insights and Experiences Gained from the Test Network}

Various applications like broadcasting of emergency warnings as well as unicast multihop communication between two vehicles have been successfully tested and demonstrated [11]. Since all these applications were below the saturation of the shared medium, we are now quantitatively evaluating the maximally achievable performance of the router in combination with the 802.11 MAC. As in [12] we are interested in assessing the achievable multi-hop throughput as well as observed latency for a chain-like topology with chain lengths 2 to 6 . We have conducted experiments in static and mobile settings for UDP and TCP traffic. Contrary to simulation results as in [12] we have observed degradation of the achievable throughput due to highly changing link conditions and due to obstacles that aggravate the hidden terminal problem. The statistical analysis and evaluation of the measurements is underway. Due to the highly changing radio conditions, in particular in mobile scenarios, simple averages over several runs do not lead to meaningful results without a proper analysis of the 'third variables' of the experiments. For example, when a node receives a beacon from a far away node to which communication is only 'occasionally' feasible, the forwarding of packets might be seriously affected. Preliminary results for the static chain topology show, e.g., for the case of a chain length of 4 nodes, IEEE 802.11 with $2 \mathrm{Mbps}$, and bidirectional communication between the two outer nodes, that a throughput of $200 \mathrm{kbps}$ and round-trip-times varying between several hundred milliseconds and 3 seconds can be achieved.

\section{Outlook}

The movements, connectivity graphs and radio conditions are all recorded during the test runs in addition to the actual measurements on throughput and latency. These data 
allow for 'replay' within a simulator, and will help us to compare simulation results with reality and to enhance simulations. While the amount of work in setting up tests with a vehicular ad hoc networks of six cars is already considerable, we plan to increase the number of cars to several tens of vehicles in order to get insight into multi-hop communication behaviour over larger areas. In addition, we plan to migrate to $802.11 \mathrm{a}$ NICs as the current ASTM standardisation process has selected 802.11a as a basis for its standard E2213-02 [13]. On the routing side, we will further investigate geocast routing strategies and will perform simulations of a new position-based forwarding strategy, called contention-based forwarding [14], on top of realistic vehicle movement patterns.

\section{References}

1. Franz, W., Eberhardt, R., Luckenbach, T.: Fleetnet - internet on the road. In: Proceedings of the 8th World Congress on Intelligent Transportation Systems. (2001) Sidney, Australia.

2. Hartenstein, H., Bochow, B., Ebner, A., Lott, M., Radimirsch, M., Vollmer, D.: Position-aware ad hoc wireless networks for inter-vehicle communications: The FleetNet project. In: Proc. ACM MobiHoc '01, Long Beach, California (2001)

3. The FleetNet project. http://www.fleetnet.de

4. Maltz, D.A., Broch, J., Johnson, D.B.: Experiences designing and building a multi-hop wireless ad hoc network testbed. Technical Report CMU-CS-99-116, School of Computer Science, Carnegie Mellon University (1999)

5. Singh, J.P., Bambos, N., Srinivasan, B., Clawin, D.: Wireless LAN Performance under Varied Stress Conditions in Vehicular Traffic Scenarios. In: Proceedings of the IEEE 56th Vehicular Technology Conference (VTC'02 Fall). Volume 2., Vancouver, Canada (2002) 743-747

6. Mauve, M., Widmer, J., Hartenstein, H.: A Survey on Position-Based Routing in Mobile Ad-Hoc Networks. IEEE Network 15 (2001) 30-39

7. Käsemann, M., Hartenstein, H., Füßler, H., Mauve, M.: A Simulation Study of a Location Service for Position-Based Routing in Mobile Ad Hoc Networks. Technical Report TR-07002, Department of Computer Science, University of Mannheim (2002)

8. Füßler, H., Mauve, M., Hartenstein, H., Käsemann, M., Vollmer, D.: A Comparison of Routing Strategies for Vehicular Ad Hoc Networks. Technical Report TR-02-003, Department of Computer Science, University of Mannheim (2002)

9. Lochert, C., Hartenstein, H., Tian, J., Füßler, H., Herrmann, D., Mauve, M.: A Routing Strategy for Vehicular Ad Hoc Networks in City Environments. In: Proc. IEEE Intelligent Vehicles Symposium, Columbus, OH (2003)

10. Karp, B.N., Kung, H.T.: GPSR: Greedy Perimeter Stateless Routing for Wireless Networks. In: Proc. ACM/IEEE MobiCom '00, Boston, Massachusetts (2000) 243-254

11. Enkelmann, W.: Fleetnet - applications for inter-vehicle communications. In: Proc. IEEE Intelligent Vehicles Symposium. (2003)

12. Li, J., Blake, C., DeCouto, D.S.J., Lee, H.I., Morris, R.: Capacity of Ad Hoc Wireless Networks. In: Proc. ACM/IEEE MobiCom '01, Rome, Italy (2001) 61-69

13. Standard specification for telecommunications and information exchange between roadside and vehicle systems. ASTM Designation: E 22123 - 02e1.

14. Füßler, H., Widmer, J., Käsemann, M., Mauve, M., Hartenstein, H.: Contention-based Forwarding for Mobile Ad Hoc Networks. Accepted for publication in the Elsevier Ad Hoc Networks Journal (2003) 